\title{
Episodic occurrence of field-aligned energetic ions on the dayside
}

\author{
Chao Yue ${ }^{1,2}$, Jacob Bortnik², Shasha Zou ${ }^{3}$, Yukitoshi Nishimura ${ }^{4}$, John C. Foster ${ }^{5}$, \\ Thomas Coppeans ${ }^{3}$, Qianli Ma²,4, Qiugang Zong ${ }^{1}$, A. J. Hull', Mike Henderson \\ Geoffrey D. Reeves ${ }^{7,8}$, Harlan E. Spence ${ }^{9}$
}

1. Institute of Space Physics and Applied Technology, Peking University, Beijing, China

2. Department of Atmospheric and Oceanic Sciences, UCLA, Los Angeles, CA, USA.

3. Climate and Space Sciences and Engineering, University of Michigan, Ann Arbor, MI, USA

4. Center for Space Physics, Boston University, Boston, MA, USA

5. Haystack Observatory, Massachusetts Institute of Technology, Westford, Massachusetts, USA

6. Space Sciences Laboratory, University of California, Berkeley, CA, USA

7. Space Science and Applications Group, Los Alamos National Laboratory, Los Alamos, NM, USA.

8. Space Sciences Division at the New Mexico Consortium, Los Alamos, NM, USA.

9. Institute for the Study of Earth, Oceans, and Space, University of New Hampshire, Durham, New Hampshire, USA.

This is the author manuscript accepted for publication and has undergone full peer review but has not been through the copyediting, typesetting, pagination and proofreading process, which may lead to differences between this version and the Version of Record. Please cite this article as doi: 10.1029/2019GL086384

This article is protected by copyright. All rights reserved. 


\begin{abstract}
The tens of $\mathrm{keV}$ ion observed in the ring current region at $\mathrm{L} \sim 3-7$, generally have pancake pitch angle distributions (PADs), that is, peaked at $90^{\circ}$. However, in this study, by using the Van Allen Probe observations on the dayside, unexpectedly we have found that about 5\% time, protons with energies of $~ 30$ to $50 \mathrm{keV}$ show two distinct populations, having an additional field-aligned population overlapping with the original pancake population. The newly appearing field-aligned population have higher occurrence rates at 12-16 MLT during geomagnetically active times. In particular, we have studied eight such events in detail and found that the source regions are located around 12 to 18 MLT which coincides with our statistical result. Based on the ionospheric and geosynchronous observations, it is suggested that these energetic ions with field-aligned PADs probably are accelerated near post-noon in association with ionospheric disturbances that are triggered by tail injections.
\end{abstract}

Plain Language Summary: Protons of different sources have different pitch angle distributions (PADs). For example, warm plasma cloak protons, which come directly from the ionosphere, have field-aligned PADs while ring current protons that generally 
originate from tail plasma sheet, have pancake-shaped PADs. In this study, unexpectedly we have found that about $5 \%$ of the time on the dayside, protons of ring current energies show two distinct populations according to their PADs: higher fluxes of field-aligned populations overlapping with the original pancake populations. The newly appeared field-aligned populations have higher occurrence rates at $12-16$ MLT during geomagnetically active times. In order to find the mechanism that generates these fieldaligned energetic proton populations, we have studied eight such events in detail by using the low altitude DMSP, POES satellites and the NOAA LANL satellite at the geosynchronous orbit. The results imply that these energetic ions with field-aligned PADs probably are accelerated by ionospheric disturbances that are triggered by tail injections. These results provide evidence of another possibly important source of the ring current ions.

\section{Key Points:}

1. We have found that about $5 \%$ of the time on the dayside, protons with energies of $\sim 30$ to $50 \mathrm{keV}$ have strong field-aligned PADs.

2. The field-aligned PADs have higher occurrence rates at $12-16$ MLT during geomagnetically active times

3. These energetic field-aligned ions possibly are accelerated by ionospheric disturbances triggered by tail injections. 


\section{Introduction}

The tens of $\mathrm{keV}$ ion populations predominantly observed in the ring current region at $\mathrm{L} \sim 3$ 3- 7, generally have pancake pitch angle distributions (PADs) (e.g., Lui, 1996; Chen et al., 1998; 1999; Yue et al., 2017), that is, fluxes peak at $\sim 90^{\circ}$ to the background magnetic field. These pancake PADs are formed due to a combination of betatron and Fermi acceleration when they are transported from the tail plasma sheet, where the major ring current plasma originates. The main charge carriers of the ring current are ions of various species, including light protons $\left(\mathrm{H}^{+}\right)$and heavy oxygen ions 
$\left(\mathrm{O}^{+}\right)$with energies ranging from one to a few hundred keV (Williams, 1985; Gkioulidou et al., 2016; Yue et al., 2018; 2019; Keika et al., 2018a). During active times, the fluxes of $\mathrm{H}^{+}$with energies of $1<\mathrm{E}<40 \mathrm{keV}$ and $\mathrm{O}^{+}$with $10<\mathrm{E}<50 \mathrm{keV}$ increase significantly (e.g., Smith and Hoffman, 1973; Krimigis et al., 1985; Yue et al., 2018; 2019). These are the predominant contributors to the ring current enhancement.

Only a few observational studies have addressed the existence of field-aligned distributions of energetic ions in the inner magnetosphere. The early observations made by Shelly et al. (1976) have shown that ions flowing out of the ionosphere are observed with energies in the $\mathrm{keV}$ range, implying the existence of some ionospheric acceleration mechanism. Sheldon et al. (1998) reported one event on 15 April 1996 showing that there was a field-aligned beam of ions around $40 \mathrm{keV}$ from $\mathrm{L}=3-7$ observed by the Polar satellite. They speculated that these field-aligned ions were not convected from some distant boundary, but in situ accelerated by a parallel electric field during the time of measurement.

In general, the energy of ionospheric outflow is only several $\mathrm{eV}$ near the topside ionosphere. In order to accelerate the ions to hundreds of $\mathrm{eV}$ or even into the $\mathrm{keV}$ range, two means of acceleration have been proposed: (1) parallel electric fields (Evans, 1974; Yau et al., 1985; Newell et al., 1996; Gkioulidou et al., 2019); (2) the dispersive Alfven wave (DAWs) (Chaston et al., 2004; 2005; 2007; Hull et al., 2019). Gkioulidou et al. (2019) have observed field-aligned $\mathrm{O}^{+}$ions near the equator with energies below $\sim 1 \mathrm{keV}$ 
that directly enter the inner magnetosphere at $\mathrm{L}=3-4$ in association with strong upward field aligned current. Based on FAST observations, Chaston et al. (2004) using a DAWs model have concluded that ions could be transversely accelerated by DAWs to keV energies in a few seconds to minutes. Addressing the relative importance of these two mechanisms, Chaston et al. (2007) have combined coincident satellite measurements of fields and particles to demonstrate that $15-34 \%$ of the total energetic ion outflow might be attributed to the action of DAWs. Recently, Chaston et al. (2015; 2016) reported that DAWs could extract ions from the topside ionosphere and accelerate these ions up to 50 $\mathrm{keV}$ at $\mathrm{L}=3$ to 6.5 . When observed in the equatorial plane, these ions would appear with field-aligned PADs due to the action of the magnetic mirror force. At increasing energies, the flux increase would tend toward perpendicular pitch angles.

In this study, by using the Van Allen Probe observations in the inner magnetosphere, we have found that about $5 \%$ of the time on the dayside, there are fieldaligned proton populations with energies ranging from $\sim 30$ to $50 \mathrm{keV}$, predominantly observed in the 12-16 magnetic local time (MLT) sector and at L shell from 5 to 5.5. We have randomly selected eight such events with clear energy dispersion to analyze in detail and found that they are well-correlated with ion injections from the magnetotail and a number of ionospheric signatures, including total electron content (TEC) enhancement, stronger proton precipitation, and proton flux intensification at sub-auroral region.

\section{Dataset and methodology}

This article is protected by copyright. All rights reserved. 
The Van Allen Probes (RBSP), which consists of two identically instrumented spacecraft (probes A and B), orbit near the equator $\left(10^{\circ}\right.$ inclination) with a perigee at $\sim 1.1$ $\mathrm{R}_{\mathrm{E}}$, an apogee at $\sim 5.8 \mathrm{R}_{\mathrm{E}}$, and a period of $\sim 9 \mathrm{~h}$ (Mauk et al., 2013). Both satellites are equipped with comprehensive suites of particles and fields measurement instrumentation. Here we perform our analysis by using the released version 4 of level-3 pitch-angle dependent differential flux measurements from the Helium, Oxygen, Proton, and Electron (HOPE) Mass Spectrometer (Funsten et al., 2013) of the Energetic Particle Composition and Thermal Plasma (ECT) Suite (Spence et al., 2013) onboard Van Allen Probes (both A and B). HOPE provide the pitch-angle dependent flux of $\mathrm{H}^{+}, \mathrm{O}^{+}$, helium $\left(\mathrm{He}^{+}\right)$and electron (e $e^{-}$with energies from several $\mathrm{eV}$ to $\sim 50 \mathrm{keV}$ in 11 pitch angle bins. In this study, we have averaged the pitch-angle dependent particle fluxes with 1-min time resolution, to investigate the PAD information of energetic ions.

For our statistical study, we focus on the times when Van Allen Probes are on the dayside (6-18 MLT) and the geomagnetic latitudes of the spacecraft are less than $10^{\circ}$. To avoid the contamination from the energetic proton butterfly distributions, which often occur on the nightside (e.g., Shi et al., 2015; Yue et al., 2017), we have excluded the nightside (18-6 MLT) observations. To qualify as a field-aligned distribution, we have required that the normalized pitch-angle dependent fluxes of $\mathrm{H}^{+}$with energy from $28 \mathrm{keV}$ to $50 \mathrm{keV}$ at the $18^{\circ}$ and $162^{\circ}$ pitch angle bins should have normalized values larger than 1.1 and the $90^{\circ}$ pitch angle bin should have a value less than 0.9. Here, the normalized 
flux is the flux divided by the averaged flux over all pitch angle bins at every time increment. After automatic selection, all the events are double checked by eye to ensure that they are indeed field-aligned distributions.

\section{Results}

In this section, we first present a typical field-aligned PAD event that occurred on 15 February 2014, and follow it with a statistical survey. The case study provides an illustration of all the common features for all eight such events that we have investigated in detail. The statistical results demonstrate that the field-aligned proton PADs occur about $5 \%$ of the time on the dayside and that higher occurrence rates can be found at $~ 12$ 16 in MLT and 5-5.5 in L shell during geomagnetically active times.

Figure 1 shows the proton pitch-angle dependent differential fluxes at seven energy channels as a function of pitch angle and universal time on 15 February 2014 observed by Van Allen Probe B near the noon sector. As shown in Figure 1a, all the energy channels between $\sim 20-50 \mathrm{keV}$ show pancake PADs before 16:05 UT, and then new field-aligned populations with larger fluxes suddenly appear superimposed on the original pancake PADs after 16:05 UT. The starting times and durations are different for different energy channels with lower energy protons starting later in time and having longer time duration. This dispersion signature may be caused by the differences in particle's drift velocity for the different energy protons on different $L$ shells from their 
source region. In order to better visualize and compare the different PADs, Figure 1b shows the normalized flux which is the number flux of ions at each pitch angle bin divided by the average flux of all the pitch angle bins at one specific time. As shown in Figure 1b, there are strong field-aligned populations starting from 16:05 UT at the 52 $\mathrm{keV}$ channel and then gradually extending to lower energy channels.

Figure 2 shows the background electron density in panel (a) derived from High Frequency Receiver (HFR) spectra of the Electric and Magnetic Field Instrument Suite and Integrated Science instrument (EMFISIS) (Kletzing et al., 2013), the energy spectrum and specific anisotropy of the proton, helium and oxygen fluxes during this same event. The specific anisotropy (e.g., Chen et al., 1998; 1999; Li et al., 2010) is the anisotropy for a specific energy channel which is calculated as $A=\frac{\int_{0}^{\pi / 2} f(E, \alpha) \sin ^{3} \alpha d \alpha}{2 \int_{0}^{\pi / 2} f(E, \alpha) \cos ^{2} \alpha \sin \alpha d \alpha}-1$, where $\alpha$ is the equatorial pitch angle, $\mathrm{E}$ is the kinetic energy, and $f$ is the particle phase space density. $\mathrm{A}=0$ corresponds to pitch angle isotropy, $\mathrm{A}>0$ represents PADs peaking at $90^{\circ}$, while $\mathrm{A}<0$ indicates a PAD minimum at $90^{\circ}$. As shown in Figure 2a, Van Allen Probe $\mathrm{B}$ was inside the plasmasphere throughout the entire time interval with the background density larger than $100 \mathrm{~cm}^{-3}$. In addition, for $\mathrm{E}>20 \mathrm{keV}$ protons, which are the major particle population contributing to the ring current, their flux increases first at high energies and then gradually moves to lower energies (Figure 2b), and the high flux region is consistent with the low specific anisotropy region highlighted by the same black contours of Figure $2 \mathrm{~b}$ and 2c, indicating that these enhancements of field-aligned 
populations of energetic protons could be a significant contributor to the quiet time ring current.

The origin of these newly appearing field-aligned ion populations is not clear, since they may come from either the tail plasma sheet due to injection, or directly from the ionosphere. In order to examine the source of these energetic protons with fieldaligned PADs, we trace back to the source region and source timing according to the observed energy dispersion due to the difference in drift velocity for different energy protons at different $\mathrm{L}$ shell. Using the time of flight technique, the time and location of the source region can be estimated by a following equation (1):

$$
t-t_{0}=\frac{M L T-M L T_{0}}{24} T_{d}
$$

where $t$ and MLT are the starting time and magnetic local time (MLT) of the observed field-aligned energetic proton distributions, $t_{0}$ is the source timing, and $M L T_{0}$ is the magnetic local time of the source region, $T_{d}$ is the drift period, which is given as $T_{d}=$ $\frac{\pi q B_{0}\left(L R_{E}\right)^{2}}{3 K\left(0.35+0.15 \sin \alpha_{0}\right)}$ in a dipole magnetic field (adapted from Roederer [1970]), where $\mathrm{B}_{0}$ is the equatorial magnetic field intensity at specific $L$ shell, $K$ is the particle kinetic energy, $\mathrm{R}_{\mathrm{E}}$ is the Earth's radius and $a_{0}$ is the equatorial pitch angle. Here, the electric field in the energetic proton's drift motion is not considered since the electric field effect is relatively weak comparing with magnetic drifts for $\mathrm{E}>10 \mathrm{keV}$ protons (e.g., Yue et al., 2017a). For this event, the average source region is around $17 \mathrm{~h}$ in $\mathrm{MLT}, 65^{\circ}$ in mapped latitude of $\mathrm{L}=5.7$ using the TS04 model and the source timing is about $15 \mathrm{UT}$. 
Figure 3 shows the low altitude observations from the POES, DMSP satellites and the geosynchronous orbit observations on the nightside from NOAA-LANL satellites. It is shown that in Figure 3a, both the field-aligned (blue line) and trapped (black line) fluxes of the energetic protons from three energy channels became enhanced after the source timing around 15 UT (bottom three panels in Figure 3a) compared with those 2 hours previously (upper three panels in Figure 3a) when the POES-18 spacecraft had access to the same location near 15 MLT. In addition, there is proton flux intensification (second panel in Figure 3c) at sub-auroral region (marked by the vertical dashed line) near 15 MLT observed by DMSP-16 after the source timing compared with those before the source timing (second panel in Figure 3b). Furthermore, comparisons between the dayside TEC maps at 14:40 UT and 15:10 UT clearly showed that the TEC on the dayside became enhanced after the source timing (15:00 UT), such as regions marked by the pink arrows in the right panel of Figure 3d. Besides, the auroral oval expanded to lower latitude from DMSP SSUSI data (not shown here) after the source timing. These ionospheric observations indicate the strong ionospheric disturbance, which may produce more ionospheric outflow. On the other hand, Figure 3e shows the more intense energetic proton flux precipitation after source timing compared with those 2 hours previously observed by POES-16 at the pre-midnight sector. Meanwhile, the energetic proton fluxes obtained from NOAA-LANL satellites (LANL-01A, LANL-02A and LANL-04A) on the nightside at the geosynchronous orbit show small fluctuations near the source timing 
(marked by the vertical dashed line in Figure 3f) while there is no flux variation on the dayside (not shown), indicating that there might be injections from the tail plasma sheet, which might be related to the enhancement of the field-aligned population observed by the Van Allen Probe in the noon sector.

To better understand these episodic field-aligned energetic ion fluxes enhancement events, we searched for all the field aligned PAD events of energetic protons from October 2012 to September 2018 when Van Allen Probes were on the dayside, and we found that this type of event occurs about 5\% of the time. Figure $4 a$ shows the back-tracing result for the source locations on the equatorial plane of the eight events that we have studied in detail according to the energy dispersion due to the difference in drift velocity of different energy protons at different L shell. It is shown that the source locations are mostly around 12-18 MLT. Figures 4b-4d show the occurrence rates of field-aligned PAD events of $32.7 \mathrm{keV}$ energetic protons under different solar and geomagnetic conditions without any backward tracing. As shown in Figure 4b, the high occurrence appears around 12-16 MLT and 4.8-5.6 in L shell, indicating that 12-16 in MLT and 4.8-5.6 in L shell could be the source region, which is consistent with the result shown in Figure 4a. Meanwhile, the occurrence rate is also higher when Dst (Figure 4d) is smaller or solar wind dynamic pressure (Figure 4c) and AE index (Figure 4e) are larger, indicating that these episodic field-aligned PADs of energetic proton events usually occur during high geomagnetic activity periods. It should be noted that the all the energy 
channels of $\sim 30-50 \mathrm{keV}$ protons from the HOPE instrument show very similar statistical results to those of the $32.7 \mathrm{keV}$ protons.

\section{Discussion}

In this study, we have investigated the occurrence rates and characteristics of episodic field-aligned PADs of protons with energies of 30 to $50 \mathrm{keV}$ based on Van Allen Probe observation from 2012 to 2018 on the dayside. We have found that about 5\% of the time there are two distinct populations, separated according to their PADs: the higher fluxes of field-aligned populations overlapping with the original pancake populations. These field-aligned PADs have higher occurrence rates at $12-16$ in MLT and 4.8-5.6 in L shell during geomagnetically active times. The higher occurrence rates in MLT and L shell and back-tracing the energy-dispersed flux observations point to the source region location.

To understand where this newly appearing field-aligned population originates, we investigated multiple such unusual events in detail by combining the NOAA-LANL observations at the geosynchronous orbit and the low altitude DMSP and POES observations. We first traced back to identify the source region and timing of these events according to the energy dispersion signatures of the field-aligned PADs, which is due to the difference in drift velocities of the different energy protons at different L shells. We found that the source regions are around 12 to 18 MLT shown in Figure 4a which is 
consistent with our statistical result that the region with higher occurrence of the episodic field-aligned PADs of energetic ions on the dayside is around 12-16 MLT as shown in Figure $4 \mathrm{~b}$. In addition, we have found that all the eight events analyzed in detail show energetic proton flux fluctuation near midnight at the geosynchronous orbit and very similar ionospheric features in the post-noon sector. For example, the low altitude DMSP shows clear proton fluxes enhancement at the sub-auroral region near the dusk side as illustrated in Figure 3. The POES satellite, whenever there is available spacecraft at the dusk sector, demonstrates clear flux enhancement of energetic protons at the same region after the source timing. Meanwhile, TEC dramatically increases and the auroral oval expands to lower latitudes around the source timing. These low altitude observations imply that there are significant ionospheric disturbances that might lead to enhanced ionospheric outflow. Through an additional mechanism accelerating the outflowing ions as they propagate along the magnetic field, these ionospheric ions could have direct access to the ring current region and be observed by Van Allen Probes. To accelerate these ionospheric ions to $10 \mathrm{~s} \mathrm{keV}$, dispersive Alfven waves could be a good candidate, as proposed by Chaston et al. (2015; 2016). Here, we also have checked the in situ DAWs observations by using Van Allen Probes, however, not all the eight cases are correlated with strong DAWs, which is reasonable since the observed field-aligned events by Van Allen Probes are not always near the source region (12-16 MLT). 
On the other hand, 50-400 keV proton fluxes observed by LANL satellites at geosynchronous orbit on the nightside always show small fluctuations near the source timing for all the eight events and stronger proton precipitation after the source time also has been observed near the midnight by POES satellite whenever there is available data as illustrated in Figure 3. These observations suggest that the field-aligned dayside populations observed by Van Allen Probes might be connected to injections from the tail plasma sheet either directly or indirectly. In general, the protons have pancake PADs if they are injected from tail plasma sheet due to stronger betatron acceleration in the perpendicular direction than the parallel Femi acceleration (e.g., Lui, 1996; Nose et al., 2000; Yue et al., 2017). However, under certain circumstance (such as a weak and slow substorm), the first invariant maybe conserved but the second invariant maybe not, ions could be primarily energized in the parallel direction (Mauk and McIlwain 1975; Mauk 1986; Fok et al., 2006). Another possible mechanism could be due to drift shell splitting (Roederer, 1967; Sibeck et al., 1987; Takahashi et al., 1997). When the ions are injected from the tail plasma sheet near midnight, they drift westward to the dayside with higher pitch angle ions drifting faster and further away from the Earth to higher L shells and lower pitch angle ions drifting slower and are closer to the Earth. However, for the events we have studied, we did not find the appropriate signatures showing the pitch angle of energetic ions increasing as the satellite moves to higher L shell.

This article is protected by copyright. All rights reserved. 
Based on our event study and statistical results, we propose that the nightside injections may trigger some ionospheric disturbances, such as stronger precipitations, total electron content (TEC) enhancement, and the proton fluxes intensification at subauroral region near dusk and the aurora oval expansion. With the intense disturbances in the ionosphere, DAW may be generated to accelerate protons to $10 \mathrm{~s} \mathrm{keV}$ along the field line when they bounce and drift across the dusk side. Our following study would focus on events near the dusk side source region to find out whether there are DAWs present in the dusk side plume region that are associated with tail injections.

\section{Summary}

In this study, by using the Van Allen Probes observations from 2012 to 2018 on the dayside, we unexpectedly have found that about $5 \%$ of the time, protons with energies of $\sim 30$ to $50 \mathrm{keV}$ show two distinct populations separated according to their PADs: the higher fluxes of field-aligned populations overlapping with the original pancake populations. The field-aligned PADs have higher occurrence rates at 12-16 MLT during geomagnetic active times. In particular, we have studied eight such events in detail and traced back the particles to their source regions according to the energy dispersion signatures of the field-aligned PADs caused by the difference in drift velocities for different energy protons at different L shell. We found that the source regions are located around 12 to 18 MLT, which is consistent with our statistical results. Based on the 
ionospheric and LANL geosynchronous observations of these eight events, it is suggested that these energetic ions with field-aligned PADs most probably are accelerated in the post-noon sector in association with ionospheric disturbances that are triggered by tail injection. Acceleration of enhanced ion outflow by DAW, as suggested by Chaston et al. (2016), is a likely mechanism. Though there are multi-point satellite observations, it is still impossible to determine where these field-aligned energetic protons originate, we leave this as an open question for future studies.

\section{Acknowledgments}

This work was supported by NASA awards NNX16AG21G (HTIDeS), NNX14AI18G, RBSP-ECT and EMFISIS funding provided by JHU/APL contract No. 967399 and 921647 under NASA's prime contract No. NAS5-01072. We acknowledge use of Van Allen Probes data of the Level 3 HOPE pitch-angle dependent particle flux data obtained from the RBSP-ECT website (https://rbspect.lanl.gov/data_pub/rbspa/hope/level3/pitchangle/), made publicly available through NASA prime contract number NAS5-01072; the Level 3 RBSPICE pitch-angle dependent particle flux data obtained from the RBSPICE website (http://rbspicea.ftecs.com/Level_3PAP/). The DMSP and TEC data is from the Madrigal database (http://cedar.openmadrigal.org/list/) and we acknowledge Dr. Anthea Coster for making TEC data available. The NOAA POES were obtained from https://www.ngdc.noaa.gov/stp/satellite/poes/dataaccess.html. The LANL data could be 
found on https://doi.org/10.5281/zenodo.3594991. We thank the Space Physics Data Facility at the NASA Goddard Space Flight Center for providing the OMNI data (https://cdaweb.gsfc.nasa.gov/index.html/).

\section{References:}

Chaston, C. C., J. W. Bonnell, C. W. Carlson, J. P. McFadden, R. E. Ergun, R. J. Strangeway, and E. J. Lund (2004), Auroral ion acceleration in dispersive Alfve' $n$ waves, J. Geophys. Res., 109, A04205, doi:10.1029/2003JA010053.

Chaston, C. C., et al. (2005), Energy deposition by Alfve'n waves into the dayside auroral oval: Cluster and FAST observations, J. Geophys. Res., 110, A02211, doi:10.1029/2004JA010483.

Chaston, C. C., C. W. Carlson, J. P. McFadden, R. E. Ergun, and R. J. Strangeway (2007), How important are dispersive Alfve'n waves for auroral particle acceleration?, Geophys. Res. Lett., 34, L07101, doi:10.1029/2006GL029144.

Chaston, C. C., J. W. Bonnell, J. R. Wygant, C. A. Kletzing, G. D. Reeves, A. Gerrard, L. Lanzerotti, and C. W. Smith (2015), Extreme ionospheric ion energization and electron heating in Alfvén waves in the storm time inner magnetosphere, Geophys. Res. Lett., 42, 10,531-10,540, doi:10.1002/2015GL066674.

This article is protected by copyright. All rights reserved. 
Chaston, C. C., J. W. Bonnell, G. D. Reeves, and R. M. Skoug (2016), Driving ionospheric outflows and magnetospheric $\mathrm{O}^{+}$energy density with Alfvén waves, Geophys. Res. Lett., 43, 4825-4833, doi:10.1002/2016GL069008.

Chen, M. W., J. L. Roeder, J. F. Fennell, and L. R. Lyons (1998), Simulations of ring current proton pitch angle distributions, J. Geophys. Res., 103, 165-178.

Chen, M. W., J. L. Roeder, J. F. Fennell, L. R. Lyons, R. L. Lambour, and M. Schulz (1999), Proton ring current pitch angle distributions: Comparison of simulations with CRRES observations, J. Geophys. Res., 104(A8), 17,379-17,389, doi:10.1029/1999JA900142.

Evans, D. S. (1974), Precipitation electron fluxes formed by a magnetic field-aligned potential difference, J. Geophys. Res., 79, 2853.

Fok, M.-C., T. E. Moore, P. C. Brandt, D. C. Delcourt, S. P. Slinker, and J. A. Fedder (2006), Impulsive enhancements of oxygen ions during substorms, J. Geophys. Res., 111, A10222, doi:10.1029/2006JA011839.

Funsten, H. O., Skoug, R. M., Guthrie, A. A., MacDonald, E. A., Baldonado, J. R., Harper, R. W., et al. (2013). Helium, Oxygen, Proton, and Electron (HOPE) mass spectrometer for the Radiation Belt Storm Probes Mission. Space Science Reviews, 179, 423-484. https://doi.org/ 10.1007/s11214 - 013 - 9968 - 7

Gkioulidou, M., Ohtani, S., Ukhorskiy, A. Y., Mitchell, D. G., Takahashi, K., Spence, H. E., et al. (2019). Low energy $(<\mathrm{keV}) \mathrm{O}+$ outflow directly into the inner magnetosphere: 
Van Allen Probes Observations. Journal of Geophysical Research: Space Physics, 124, 405-419. https://doi.org/10.1029/2018JA025862

Gkioulidou, M., Ukhorskiy, A. Y., Mitchell, D. G., \& Lanzerotti, L. J. (2016). Storm time dynamics of ring current protons: Implications for the long - term energy budget in the inner magnetosphere. Geophysical Research Letters, 43, 4736 - 4744. https://doi.org/10.1002/ 2016GL068013

Hull, A. J., Chaston, C. C., Bonnell, J. W., Wygant, J. R., Kletzing, C. A., Reeves, G. D., \& Gerrard, A. (2019). Dispersive Alfvén wave control of O+ ion outflow and energy densities in the inner magnetosphere. Geophysical Research Letters, 46, 8597-8606. https://doi.org/ 10.1029/2019GL083808

Keika, K., Kasahara, S., Yokota, S., Hoshino, M., Seki, K., Nosé, M., et al. (2018). Ion energies dominating energy density in the inner magnetosphere: Spatial distributions and composition, observed by Arase/MEP - i. Geophysical Research Letters, 45, 12,15312,162. https://doi.org/10.1029/2018GL080047

Kletzing, C. A., Kurth, W.S., Acuna, M., MacDowall, R.J., Torbert, R.B., Averkamp, T., Bodet, D., Bounds, S.R., Chutter, M., Connerney, J. and Crawford, D. (2013), The Electric and Magnetic Field Instrument Suit and Integrated Science (EMFISIS) on RBSP, Space Sci. Rev., doi:10.1007/s11214-013-9993-6.

Krimigis, S. M., McEntire, R. W., Potemra, T. A., Gloeckler, G., Scarf, F. L., \& Shelley, E. G. (1985). Magnetic storm of September 4, 1984 - A synthesis of ring current spectra 
and energy densities measured with AMPTE/CCE. Geophysical Research Letters (ISSN 0094-8276), 12, 329-332. http://doi.org/10.1029/GL012i005p00329.

Li, W., et al. (2010), THEMIS analysis of observed equatorial electron distributions responsible for the chorus excitation, J. Geophys. Res., 115, A00F11, doi:10.1029/2009JA014845.

Lui, A. T. Y. (1996), Current disruption in the Earth’s magnetosphere: Observations and models, J. Geophys. Rev., 101, 13,067-13,088.

Mauk, B. H. (1986), Quantitative modeling of the “convection surge”' mechanism of ion acceleration, J. Geophys. Res., 91, 13,423-13,431.

Mauk, B. H., and C. E. McIlwain (1975), UCSD auroral particles experiment, IEEE Trans. Aerosp. Electron. Syst., AES-11, 1125-1130.

Mauk, B. H., Fox, N. J., Kanekal, S. G., Kessel, R. L., Sibeck, D. G., \& Ukhorskiy, A. (2013). Science objectives and rationale for the Radiation Belt Storm Probes Mission. Space Science Reviews, 179, 3-27. https://doi.org/10.1007/s11214 - 012 - 9908 - y

Newell, P. T., K. M. Lyons, and C.-I. Meng (1996), A large survey of electron acceleration events, J. Geophys. Res., 101, 2599.

Nose, M., A. T. Y. Lui, S. Ohtani, B. H. Mauk, R. W. McEntire, D. J. Williams, T. Mukai, and K. Yumoto (2000), Acceleration of oxygen ions of ionospheric origin in the near-Earth magnetotail during substorms, J. Geophys. Res., 105, 7669-7677.

This article is protected by copyright. All rights reserved. 
Roederer, J. G. (1967). On the adiabatic motion of energetic particles in a model magnetosphere. Journal of Geophysical Research, 72(3), 981-992.

Roederer, J. G. (1970), Dynamics of geomagnetically Trapped radiation, Springer-Verlag, New York.

Sheldon, R. B., Spence, H. E., \& Fennell, J. F. (1998). Observation of the $40 \mathrm{keV}$ field aligned ion beams. Geophysical research letters, 25(10), 1617-1620.

Shelley, E. G., Sharp, R. D., \& Johnson, R. G. (1976). Satellite observations of an ionospheric acceleration mechanism. Geophysical Research Letters, 3(11), 654-656.

Shi, R., Summers, D., Ni, B., Manweiler, J. W., Mitchell, D. G., \& Lanzerotti, L. J. (2016). A statistical study of proton pitch angle distributions measured by the radiation belt storm probes ion composition experiment. Journal of Geophysical Research: Space Physics, 121, 5233-5249. https://doi.org/10.1002/2015JA022140

Sibeck, D. G., McEntire, R. W., Lui, A. T. Y., Lopez, R. E., \& Krimigis, S. M. (1987). Magnetic field drift shell splitting: Cause of unusual dayside particle pitch angle distributions during storms and substorms. Journal of Geophysical Research: Space Physics, 92(A12), 13485-13497.

Smith, P.H. and R.A. Hoffman (1973). Ring current particle distributions during the magnetic storms of December 16-18, 1971, J. Geophys. Res. 78(2), 4731-. http://doi.org/10.1029/JA078i022p04731. 
Spence, H. E., Reeves, G. D., Baker, D. N., Blake, J. B., Bolton, M., Bourdarie, S., et al. (2013). Science goals and overview of the radiation belt storm probes (RBSP) energetic particle, composition, and thermal plasma (ECT) suite on NASA's Van Allen probes mission. Space Science Reviews, 179, 311-336. https://doi.org/10.1007/s11214 - 013 $0007-5$

Takahashi, K., Anderson, B. J., Ohtani, S. I., Reeves, G. D., Takahashi, S., Sarris, T. E., \& Mursula, K. (1997). Drift - shell splitting of energetic ions injected at pseudo substorm onsets. Journal of Geophysical Research: Space Physics, 102(A10), 2211722130.

Williams, D. J. (1985). Dynamic of the Earth's ring current: Theory and observation. Space Science Reviews, 42(3 - 4), 375-396. https://doi. org/10.1007/bf00214994

Yau, A. W., E. G. Shelley, W. K. Peterson, and L. Lenchyshyn (1985), Energetic auroral and polar ion outflow at DE 1 altitudes: Magnitude, composition, magnetic activity dependence, and long-term variations, J. Geophys. Res., 90, 8417.

Yue C. et al (2017), The characteristic pitch angle distributions of $1 \mathrm{eV}$ to $600 \mathrm{keV}$ protons near the equator based on Van Allen Probes observations, J. Geophys. Res. Space Physics, 122, doi:10.1002/2017JA024421.

Yue, C., J. Bortnik, L. Chen, Q. Ma, R. M. Thorne, G. D. Reeves, and H. E. Spence (2017a), Transitional behavior of different energy protons based on Van Allen Probes observations, Geophysical Research Letters, 44, doi:10.1002/2016GL071324.

This article is protected by copyright. All rights reserved. 
Yue, C., Bortnik, J., Li, W., Ma, Q., Gkioulidou, M., Reeves, G. D., et al. (2018). The composition of plasma inside geostationary orbit based on Van Allen Probes observations. Journal of Geophysical Research: Space Physics, 123, 6478-6493. https://doi.org/10.1029/2018JA025344

Yue, C., Bortnik, J., Li, W., Ma, Q., Wang, C. - P., Thorne, R. M., et al (2019). Oxygen Ion Dynamics in the Earth's Ring Current: Van Allen Probes Observations. Journal of Geophysical Research: Space Physics, 124. https://doi.org/10.1029/2019JA026801

\section{Figure Captions:}

Figure 1. The pitch-angle dependent differential fluxes of protons with different energies as a function of pitch angle and universal time on 15 February 2014. (a) The number flux; (b) the normalized flux to better visualize and compare the PADs.

Figure 2. (a) The background electron density suggesting that the observation is in the plasmasphere; (b) The energy spectrum, and (c) the specific anisotropy of the proton, helium and oxygen ion populations. The over-plotted black lines are the contours of constant number fluxes to highlight the strong flux region. 
Figure 3. (a) The energetic proton fluxes observations by POES-18 near dusk at low altitude: upper three panels from 13:48 to 13:56 UT; bottom three panels from 15:28 to 15:36 UT. The black line represents the trapped population while the blue line represents the precipitating population. (b and c) DMSP 16 observation of electron and proton fluxes and velocity at low altitude from 13:34 to 13:40 UT (b) and from 15:16 to 15:19 UT (c). The vertical dashed lines mark the high latitude boundary of sub-auroral region. (d) the total electron content distributions at 14:40 UT and 15:10 UT. The pink dot marks the Van Allen Probe projection on the ionosphere. (e) The energetic proton fluxes observations by POES-16 near pre-midnight at low altitude: upper three panels from 13:27 to $13: 30 \mathrm{UT}$; bottom three panels from 15:10 to 15:13 UT. The black line represents the trapped population while the blue line represents the precipitating population. (f) The nightside energetic proton fluxes observations made by NOAALANL satellites at the geosynchronous orbit. Different color lines represent different energy channels as labeled on the right. The red vertical dashed line marks the source time at 15:00 UT and the solid lines mark different MLTs with green representing 6 MLT; black is 24 MLT and blue is 18 MLT.

Figure 4. (a) The back-tracing results in the MLT and L-shell plane for eight fieldaligned PAD events according to the energy dispersion due to the difference in drift velocity of different energy protons. Different colors represent different events. (b)-(e) 
The occurrence rate distributions of the $32.7 \mathrm{keV}$ proton field-aligned PADs on the dayside based on Allen Probe observations from 2012-2018. (b) MLT vs L plane; (c) Solar wind dynamic pressure vs L plane; (d) the Dst index vs L plane; (e) AE index vs L plane. 
(a) HOPE_P_ScPotCorr_140215_B_\#flux $\left(\mathrm{s}^{-1} \mathrm{~cm}^{-2} \mathrm{ster}^{-1} \mathrm{keV}^{-1}\right)$
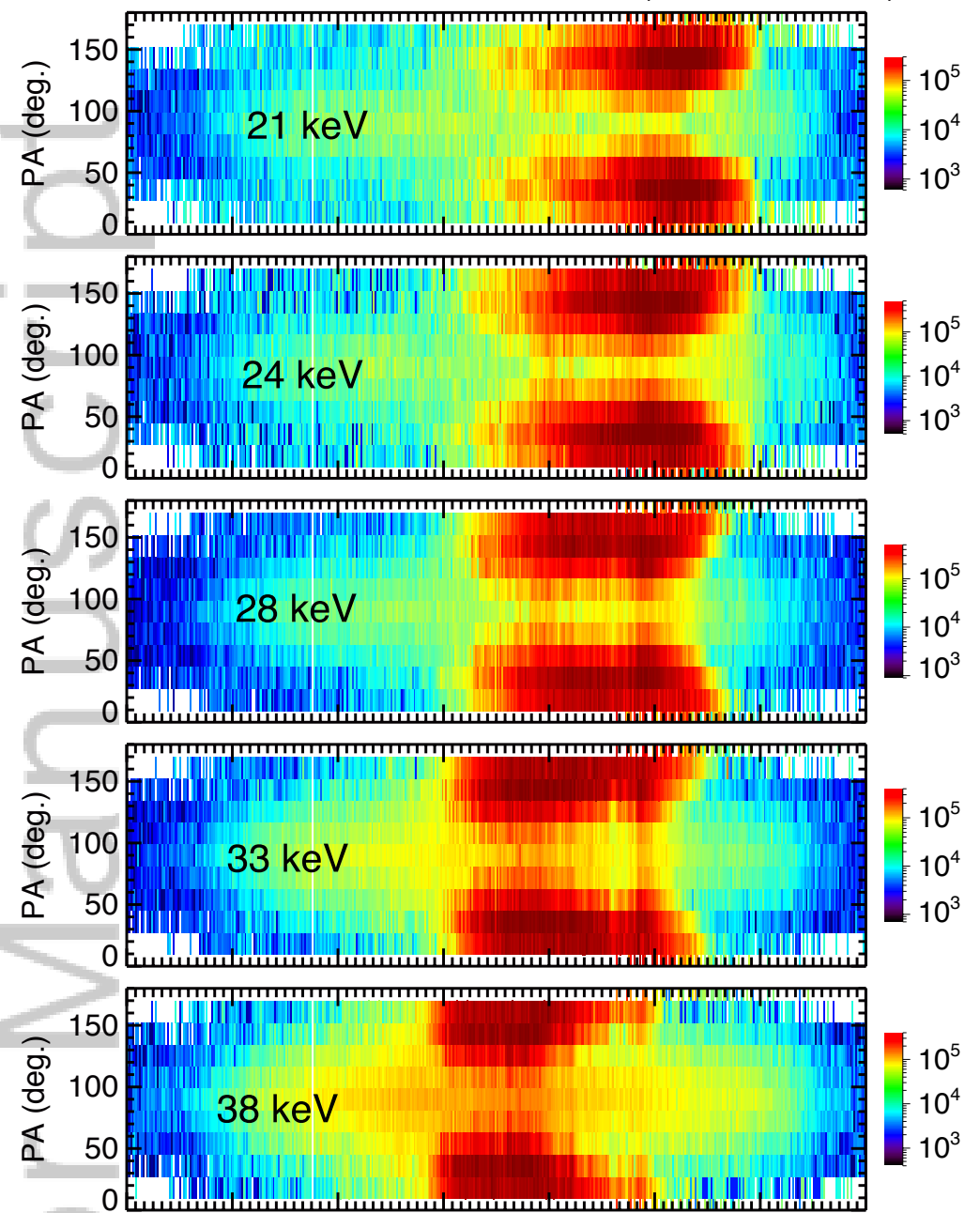

$$
8
$$
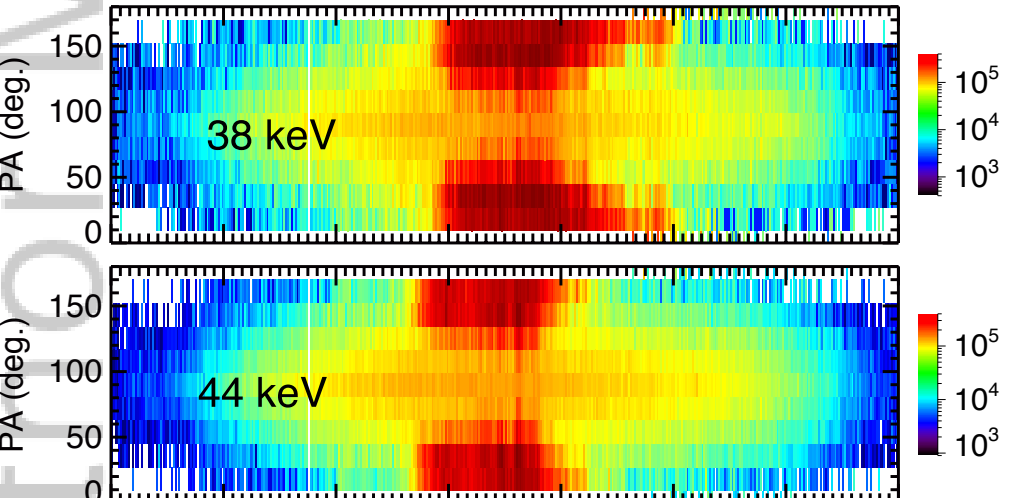

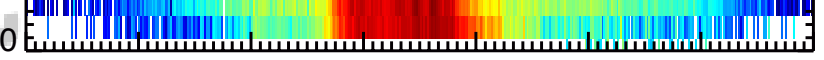
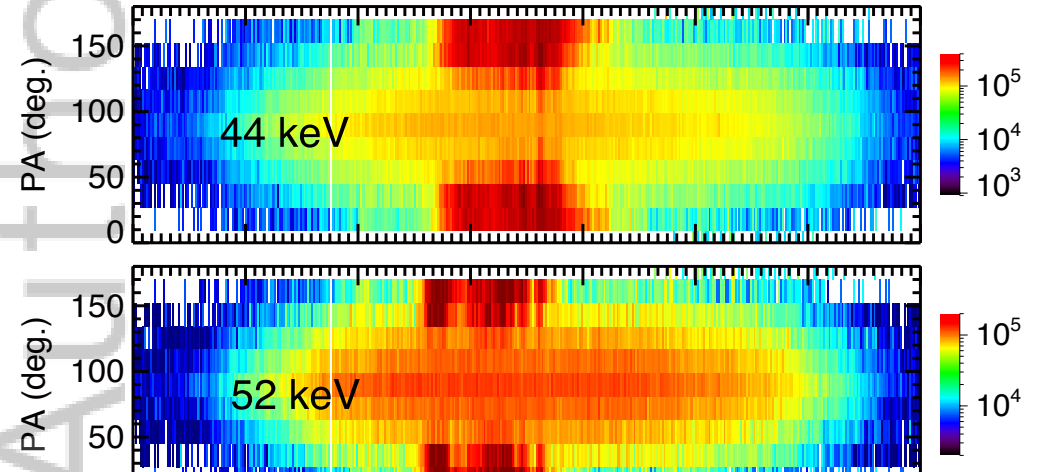

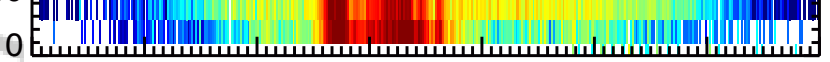

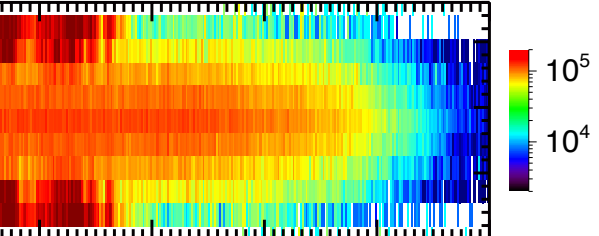

$\begin{array}{llllllll}13: 30 & 14: 30 & 15: 30 & 16: 30 & 17: 30 & 18: 30 & 19: 30 & 20: 30\end{array}$

$\begin{array}{llllllll}5: 31 & 10: 04 & 11: 04 & 11: 49 & 12: 30 & 13: 12 & 14: 04 & 15: 29\end{array}$

$\begin{array}{llllllll}7.59 & -0.37 & -0.32 & 0.27 & 1.11 & 2.24 & 3.98 & 7.50\end{array}$

$\begin{array}{llllllll}3.24 & 4.27 & 5.24 & 5.72 & 5.79 & 5.44 & 4.64 & 3.26\end{array}$

(b) HOPE P ScPotCorr 140215 B flux ratio
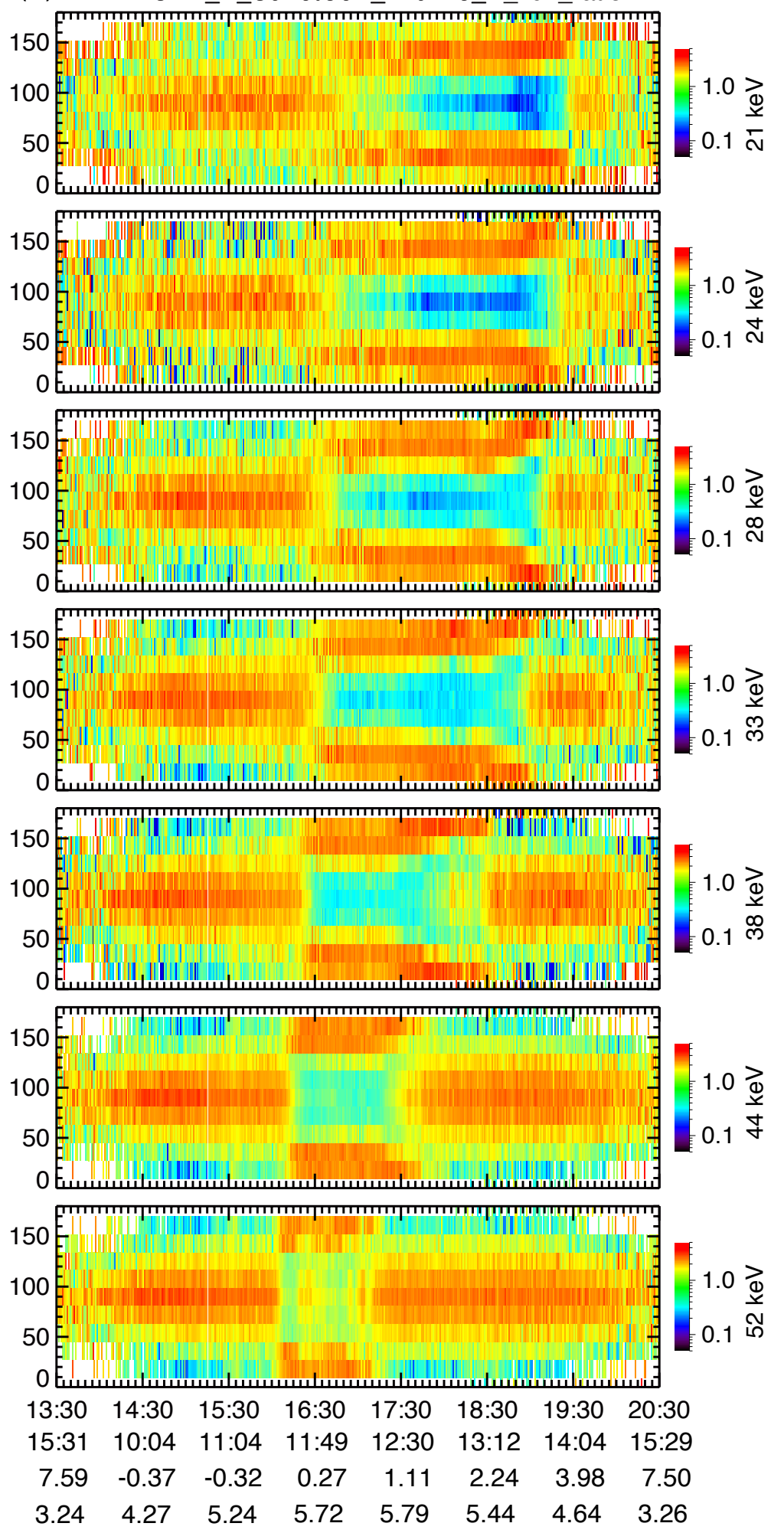


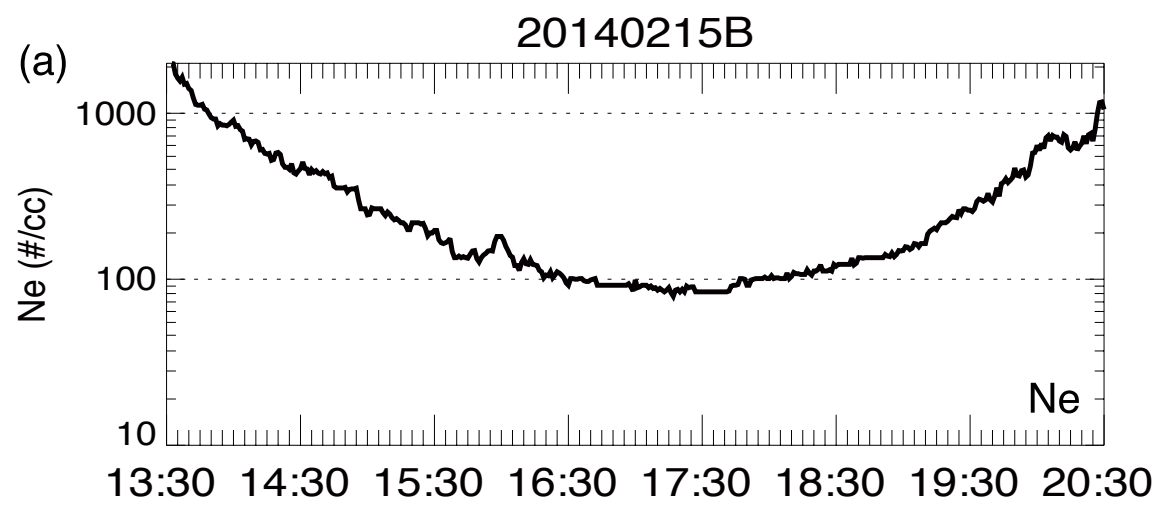

(b)
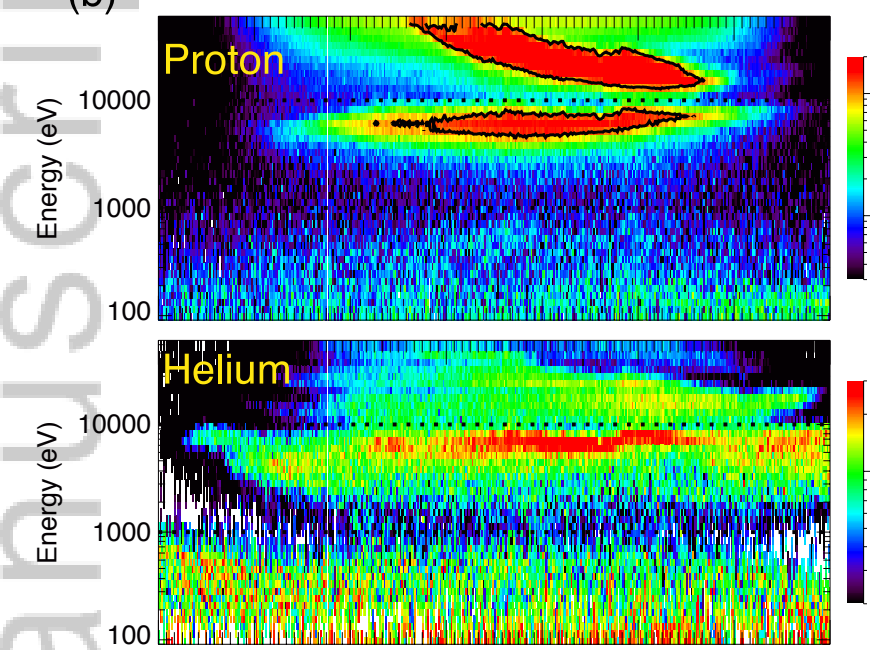

sim

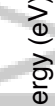

के

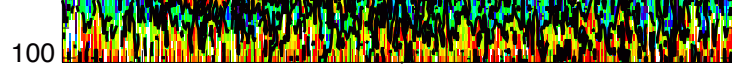

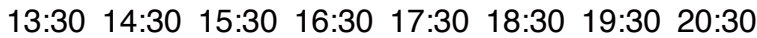

(c)

RBSP-B: Anisotropy
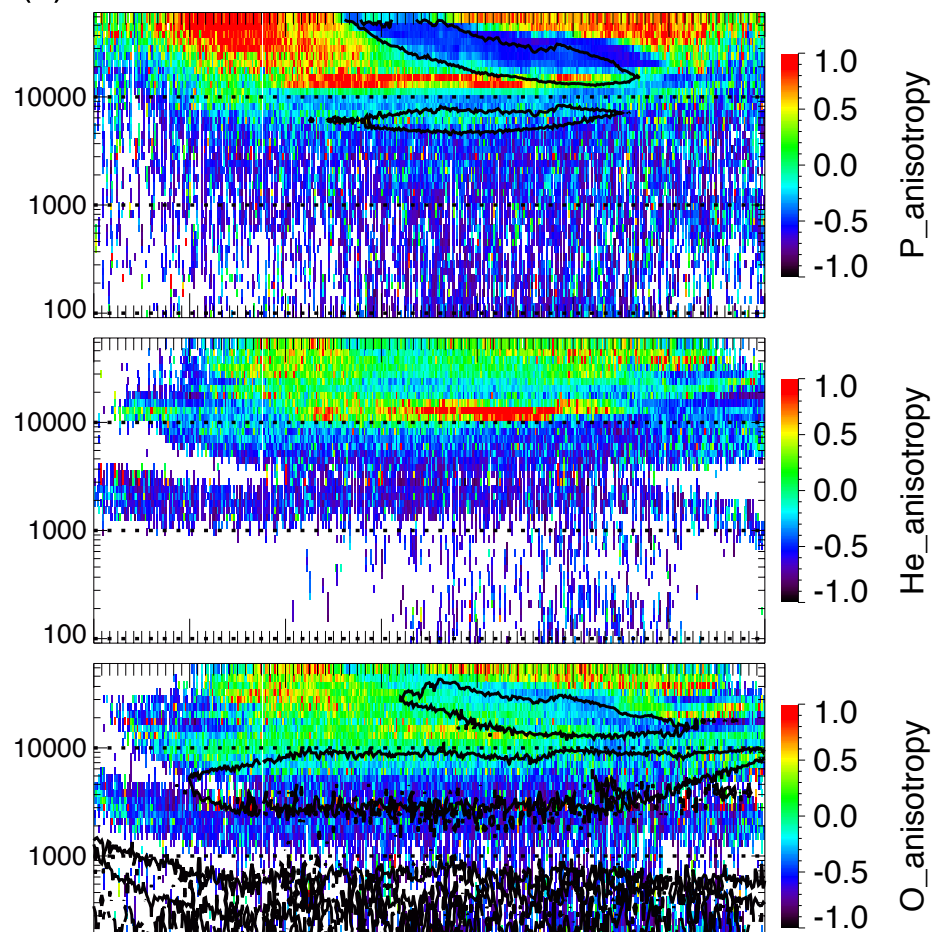

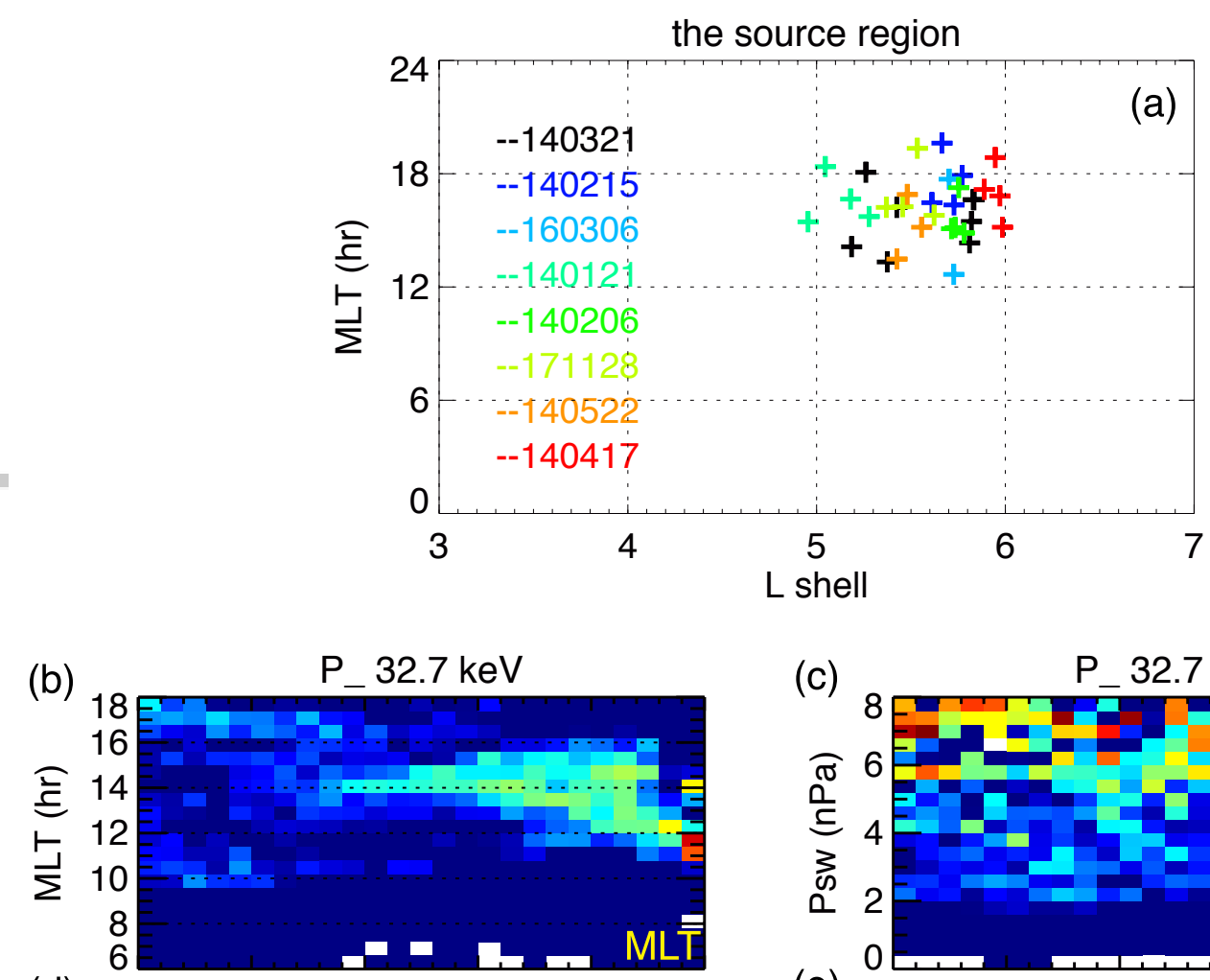

(d)
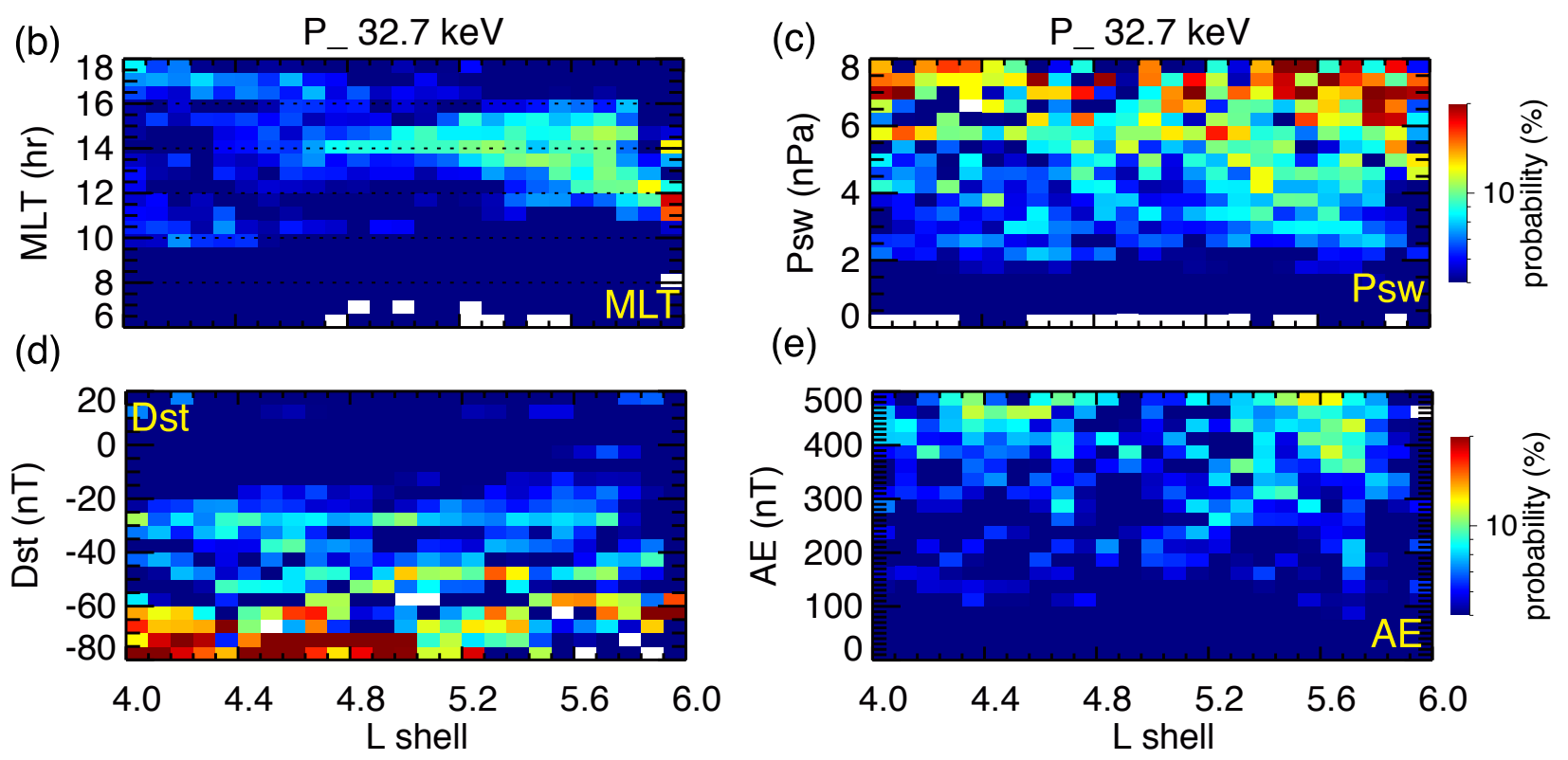

This article is protected by copyright. All rights reserved. 